Pré-Publicações do Departamento de Matemática

Universidade de Coimbra

Preprint Number 08-26

\title{
FINITE DIFFERENCE APPROXIMATIONS FOR A FRACTIONAL ADVECTION DIFFUSION PROBLEM
}

\author{
ERCÍLIA SOUSA
}

\begin{abstract}
The use of the conventional advection diffusion equation in many physical situations has been questioned by many investigators in recent years and alternative diffusion models have been proposed. Fractional space derivatives are used to model anomalous diffusion or dispersion, where a particle plume spreads at a rate inconsistent with the classical Brownian motion model. When a fractional derivative replaces the second derivative in a diffusion or dispersion model, it leads to enhanced diffusion, also called superdiffusion. We consider a one dimensional advection-diffusion model, where the usual second-order derivative gives place to a fractional derivative of order $\alpha$, with $1<\alpha \leq 2$. We derive explicit finite difference schemes which can be seen as generalizations of already existing schemes in the literature for the advection-diffusion equation. We present the order of accuracy of the schemes and in order to show its convergence we prove they are stable under certain conditions. In the end we present a test problem.
\end{abstract}

KEYwords: Fractional advection diffusion, finite differences, stability.

\section{Introduction}

Fractional calculus is a useful mathematical tool for applied sciences and recently fractional differential equations have found new applications in engineering, physics, chemistry, hydrology and other sciences. Some of the applications are given in [1], [2], [3], [4], [5], [6] to name just a few.

Fractional derivatives are used to model anomalous dispersion or diffusion. The physical experiments demonstrating the anomalous diffusion have led to an intensive effort recently to find accurate and stable numerical methods that are also easy to implement. Some numerical schemes have been developed for diffusion problems, [7], [8], [9], [10], [11], [12] and for advection diffusion problems [13], [14]. However, the stability and convergence of numerical schemes for fractional partial differential equations need further investigations. Finite difference schemes for fractional partial differential equations are more complex than the usual finite difference schemes for partial differential equations since the fractional derivative of order $\alpha$ at a certain point $x$ is a local property only when $\alpha$ is an integer. Therefore it is expected

Received June 6, 2008. 
that the theory involves information of the function further out of the region close to the point at which we are computing the derivative. Additionally the approximations of those fractional derivatives involve a number of points that changes according to how far we are from the boundary.

In this work, we derive finite difference schemes for a fractional advection diffusion equation proposed by Benson et al [15]. Benson et al [15] use a fractional advection-dispersion equation to simulate transport processes with heavy tails and demonstrate the equivalence between these heavy-tailed motions and transport equations that use fractional-order derivatives.

The fractional advection diffusion equation proposed by Benson et al [15], and used in several applications [2], [5] [6], can be expressed as follows

$$
\frac{\partial u}{\partial t}+V \frac{\partial u}{\partial x}=D\left(\frac{1}{2}+\frac{\beta}{2}\right) \frac{\partial^{\alpha} u}{\partial x^{\alpha}}+D\left(\frac{1}{2}-\frac{\beta}{2}\right) \frac{\partial^{\alpha} u}{\partial(-x)^{\alpha}},
$$

where $u$ is the resident solute concentration, $V$ is the average pore-water velocity, $x$ is the spatial coordinate, $t$ is the time, $D$ is the diffusion coefficient, $\alpha$ is the order of the fractional differentiation with $1<\alpha \leq 2$. The parameter $\beta$ is the relative weight of solute particle forward versus backward transition probability with $-1 \leq \beta \leq 1$. For $-1 \leq \beta \leq 0$, the transition probability is skewed backward, while for $0 \leq \beta \leq 1$ the transition probability is skewed forward.

If we define a fractional operator, $\nabla_{\beta}^{\alpha}$, such as,

$$
2 \nabla_{\beta}^{\alpha} u=(1+\beta) \frac{\partial^{\alpha} u}{\partial x^{\alpha}}+(1-\beta) \frac{\partial^{\alpha} u}{\partial(-x)^{\alpha}},
$$

equation (1) can be written in a simple form

$$
\frac{\partial u}{\partial t}+V \frac{\partial u}{\partial x}=D \nabla_{\beta}^{\alpha} u
$$

The Riemann-Liouville fractional derivatives of order $\alpha$, for $x \in[a, b]$, are defined by

$$
\begin{aligned}
\frac{\partial^{\alpha} u}{\partial x^{\alpha}}(x, t) & =\frac{1}{\Gamma(n-\alpha)} \frac{\partial^{n}}{\partial x^{n}} \int_{a}^{x} \frac{u(\xi, t)}{(x-\xi)^{\alpha-n+1}} d \xi, n=[\alpha]+1, x>a,(4) \\
\frac{\partial^{\alpha} u}{\partial(-x)^{\alpha}}(x, t) & =\frac{(-1)^{n}}{\Gamma(n-\alpha)} \frac{\partial^{n}}{\partial x^{n}} \int_{x}^{b} \frac{u(\xi, t)}{(\xi-x)^{\alpha-n+1}} d \xi, n=[\alpha]+1, x<b,(5)
\end{aligned}
$$


where $\Gamma(\cdot)$ is the Gamma function. Another way to represent the fractional derivatives is by the Grünwald-Letnikov formula, that is,

$$
\begin{aligned}
\frac{\partial^{\alpha} u}{\partial x^{\alpha}}(x, t) & =\lim _{\Delta x \rightarrow 0} \frac{1}{\Delta x^{\alpha}} \sum_{k=0}^{\left[\frac{x-a}{\Delta x}\right]}(-1)^{k}\left(\begin{array}{c}
\alpha \\
k
\end{array}\right) u(x-k \Delta x, t), \\
\frac{\partial^{\alpha} u}{\partial(-x)^{\alpha}}(x, t) & =\lim _{\Delta x \rightarrow 0} \frac{1}{\Delta x^{\alpha}} \sum_{k=0}^{\left[\frac{b-x}{\Delta x}\right]}(-1)^{k}\left(\begin{array}{c}
\alpha \\
k
\end{array}\right) u(x+k \Delta x, t),
\end{aligned}
$$

where $[a]$ denotes the integer part of $a$. Properties about the fractional derivatives can be found for instance in [16], [17] and [18].

To determine our explicit schemes we use a variant of the GrünwaldLetnikov formula, in which the function evaluations are shifted to the right or left and we obtain what is called the shifted Grünwald-Letnikov formula. The shift here corresponds to replacing $u(x-k \Delta x, t)$ and $u(x+k \Delta x, t)$ by $u(x-k \Delta x+\Delta x, t)$ and $u(x+k \Delta x-\Delta x, t)$ respectively, which does not affect the limit as $\Delta x \rightarrow 0$.

The reason to choose the shifted Grünwald-Letnikov formula is twofold. In one hand this is the way to obtain generalisations of already existing schemes, that is, when $\alpha=2$ we end up with schemes that are already known in literature for the advection diffusion equation. The second reason is that the approximations of the derivatives obtained by using the classical GrünwaldLetnikov formula very frequently originate unstable numerical schemes, see for instance, [14].

The structure of the paper is as follows. In section 2, we describe the finite difference schemes. Some of the numerical schemes presented in the literature for this type of problems are implicit, but explicit schemes can be more appropriate for transient problems and are easier to implement. Our schemes are explicit and can be seen as generalisations of already existing schemes for the advection diffusion equation. In section 3 the matricial form of the numerical schemes is given. In section 4 the convergence of the numerical methods is presented. In order to prove the convergence we show the accuracy of the numerical schemes and prove they are stable under certain conditions. In section 5, we present a test problem and the numerical solution is compared with an exact solution. Finally in section 6 we write some conclusions. 


\section{Finite difference schemes}

In this section we develop finite difference schemes for the fractional advection diffusion equation (1). To derive a finite difference scheme we suppose there are approximations $\mathbf{U}^{n}:=\left\{U_{j}^{n}\right\}$ to the values $U\left(x_{j}, t_{n}\right)$ at the mesh points

$$
x_{j}=j \Delta x, j=-N, \ldots,-2,-1,0,1,2, \ldots, N \quad \text { and } \quad t_{n}=n \Delta t, n \geq 0,
$$

where $\Delta x$ denotes the uniform space step and $\Delta t$ the uniform time step. For the uniform space step $\Delta x$ and time step $\Delta t$, let

$$
\nu=\frac{V \Delta t}{\Delta x} \quad \text { and } \quad \mu_{\alpha}=\frac{D \Delta t}{\Delta x^{\alpha}} .
$$

The quantity $\nu$ is called the Courant (or CFL) number and $\mu_{\alpha}$ is associated with the diffusion coefficient.

To describe the finite difference schemes, we use the upwind, central and second difference operators, given respectively by

$\Delta_{-} U_{j}^{n}:=U_{j}^{n}-U_{j-1}^{n}, \Delta_{0} U_{j}^{n}:=\frac{1}{2}\left(U_{j+1}^{n}-U_{j-1}^{n}\right)$ and $\delta^{2} U_{j}^{n}:=U_{j+1}^{n}-2 U_{j}^{n}+U_{j-1}^{n}$.

A discrete approximation to the fractional derivative terms is defined from the shifted Grünwald-Letnikov formulae,

$$
\begin{aligned}
\left(\frac{\partial^{\alpha} u}{\partial x^{\alpha}}\right)_{j}^{n} & \simeq \frac{1}{\Delta x^{\alpha}} \sum_{k=0}^{N+j+1} g_{k} U_{j+1-k}^{n}, \\
\left(\frac{\partial^{\alpha} u}{\partial(-x)^{\alpha}}\right)_{j}^{n} & \simeq \frac{1}{\Delta x^{\alpha}} \sum_{k=0}^{N-j+1} g_{k} U_{j-1+k}^{n}
\end{aligned}
$$

for

$$
g_{k}=(-1)^{k}\left(\begin{array}{c}
\alpha \\
k
\end{array}\right)=(-1)^{k} \frac{\alpha(\alpha-1) \ldots(\alpha-k+1)}{k !}=\frac{\Gamma(k-\alpha)}{\Gamma(-\alpha) \Gamma(k+1)} .
$$

The fractional operator $\nabla_{\beta}^{\alpha}$, defined by (2), is approximated by $\delta_{\beta}^{\alpha} / 2 \Delta x^{\alpha}$ where $\delta_{\beta}^{\alpha}$ is given by

$$
\delta_{\beta}^{\alpha} U_{j}^{n}=(1+\beta) \sum_{k=0}^{N+j+1} g_{k} U_{j+1-k}^{n}+(1-\beta) \sum_{k=0}^{N-j+1} g_{k} U_{j-1+k}^{n} .
$$


A finite difference scheme to approximate (1) can therefore have the form

$$
U_{j}^{n+1}=U_{j}^{n}-\nu \Delta_{-} U_{j}^{n}+\frac{1}{2} \mu_{\alpha} \delta_{\beta}^{\alpha} U_{j}^{n} .
$$

Here, an upwind discretisation for the advective term is considered and henceforth we call this scheme the upwind scheme.

Another approximation can be obtained by replacing the upwind operator with the central operator, such as,

$$
U_{j}^{n+1}=U_{j}^{n}-\nu \Delta_{0} U_{j}^{n}+\frac{1}{2} \mu_{\alpha} \delta_{\beta}^{\alpha} U_{j}^{n} .
$$

We call this scheme the central scheme, according to the discretisation of the advective term.

The third scheme is derived in a similar way to the Lax-Wendroff scheme [19] and therefore we call this scheme Lax-Wendroff scheme. Let us expand $u$ around time level $n$ to obtain

$$
u^{n+1}=u^{n}+\Delta t \frac{\partial u}{\partial t}+\frac{\Delta t^{2}}{2} \frac{\partial^{2} u}{\partial t^{2}}+\mathcal{O}\left(\Delta t^{3}\right) .
$$

From (3),

$$
\frac{\partial^{2} u}{\partial t^{2}} \simeq V^{2} \frac{\partial^{2} u}{\partial x^{2}}
$$

where the generally small (being multiplied by $D$ ) $\alpha+1$ and higher-spatialderivative terms have been dropped from (14). Note that for $D=0$,

$$
\frac{\partial^{2} u}{\partial t^{2}}=V^{2} \frac{\partial^{2} u}{\partial x^{2}}
$$

Inserting (3) and (14) into (13) gives

$$
U_{j}^{n+1}=U_{j}^{n}+\Delta t\left[-V\left(\frac{\partial u}{\partial x}\right)_{j}^{n}+D\left(\nabla_{\beta}^{\alpha} u\right)_{j}^{n}\right]+\frac{\Delta t^{2}}{2} V^{2}\left(\frac{\partial^{2} u}{\partial x^{2}}\right)_{j}^{n} .
$$

Therefore, the Lax-Wendroff scheme is of the form

$$
U_{j}^{n+1}=U_{j}^{n}-\nu \Delta_{0} U_{j}^{n}+\frac{1}{2} \mu_{\alpha} \delta_{\beta}^{\alpha} U_{j}^{n}+\frac{1}{2} \nu^{2} \delta^{2} U_{j}^{n} .
$$




\section{Matricial form of the finite difference schemes}

All our explicit methods can be written in the form of a matrix equation. Assume the nodal points are $U_{j}^{n}, j=-N, \ldots,-1,0,1, \ldots, N$ and that the boundary conditions are given, that is, we know the function values $U_{-N}^{n}$ and $U_{N}^{n}$ for $n=0,1,2, \ldots$

Introducing the vector $\mathbf{U}^{n}=\left[U_{-N+1}^{n}, \ldots, U_{-1}^{n}, 0, U_{1}^{n}, \ldots, U_{N-1}^{n}\right]^{T}$ the schemes may be written as matrix equations

$$
\mathbf{U}^{n+1}=M \mathbf{U}^{n}+\mathbf{v}^{n}, n=0,1,2, \ldots,
$$

where $M$ is the $(2 N-1) \times(2 N-1)$ matrix iteration and $\mathbf{v}^{n}$ contains boundary values. In what follows, we write out the matrix $M$ and the vector $\mathbf{v}^{n}$, for the numerical methods of the previous section.

The matrix iteration $M$ has the form

$$
M=A+\frac{1}{2} \mu_{\alpha} B
$$

where $A$ and $B$ are matrices of dimension $(2 N-1) \times(2 N-1)$ and $A$ is related with the advection discretisations and $B$ with the diffusion discretisations.

For all the three schemes the matrix $B$ is the same and is given by

$$
B=(1+\beta) L+(1-\beta) L^{T}
$$

where

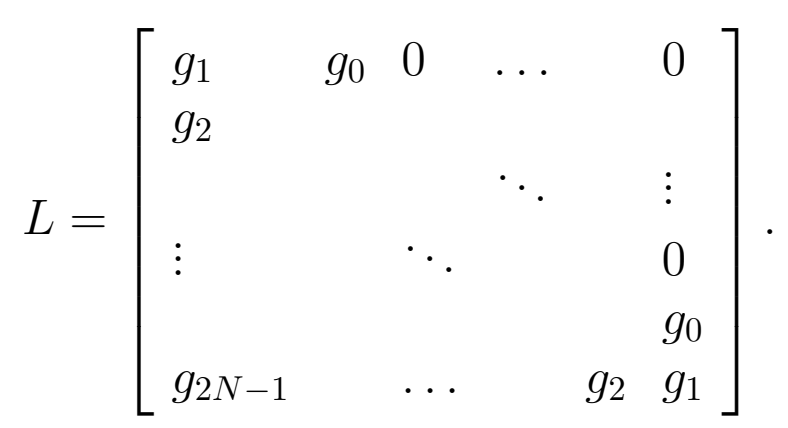

The vector $\mathbf{v}^{n}$ is composed of two parts

$$
\mathbf{v}^{n}=\mathbf{v}_{A}^{n}+\mathbf{v}_{B}^{n},
$$

where the vector $\mathbf{v}_{A}^{n}$ contains the boundary values related to the matrix $A$ and the vector $\mathbf{v}_{B}^{n}$ contains the boundary values related to the matrix $B$. For $\mathbf{v}_{B}^{n}$ we have

$$
\mathbf{v}_{B}^{n}=\mathbf{v}_{B+}^{n}+\mathbf{v}_{B-}^{n}
$$


where

$$
\mathbf{v}_{B+}^{n}=\frac{1}{2} \mu_{\alpha}(1+\beta)\left[\begin{array}{l}
g_{2} \\
\vdots \\
g_{2 N-1} \\
g_{2 N}
\end{array}\right] U_{-N}^{n}+\frac{1}{2} \mu_{\alpha}(1+\beta)\left[\begin{array}{l}
0 \\
\vdots \\
0 \\
g_{0}
\end{array}\right] U_{N}^{n}
$$

and

$$
\mathbf{v}_{B-}^{n}=\frac{1}{2} \mu_{\alpha}(1-\beta)\left[\begin{array}{l}
g_{0} \\
0 \\
\vdots \\
0
\end{array}\right] U_{-N}^{n}+\frac{1}{2} \mu_{\alpha}(1-\beta)\left[\begin{array}{l}
g_{2 N} \\
g_{2 N-1} \\
\vdots \\
g_{2}
\end{array}\right] U_{N}^{n} .
$$

For the upwind scheme the matrix $A$ and the vector $\mathbf{v}_{A}^{n}$ are given respectively by the matrix $A_{U}$ and the vector $\mathbf{v}_{A_{U}}^{n}$ defined as follows

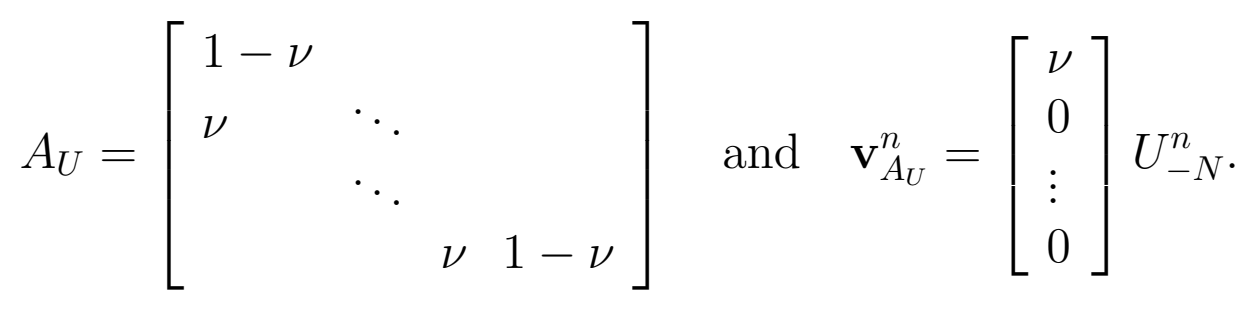

For the central scheme the matrix $A$ and the vector $\mathbf{v}_{A}^{n}$ are given respectively by the matrix $A_{C}$ and the vector $\mathbf{v}_{A_{C}}^{n}$,

$A_{C}=\left[\begin{array}{lllr}1 & -\nu / 2 & & \\ \nu / 2 & \ddots & \ddots & \\ & \ddots & & -\nu / 2 \\ & & \nu / 2 & 1\end{array}\right]$ and $\mathbf{v}_{A_{C}}^{n}=\left[\begin{array}{l}\nu / 2 \\ 0 \\ \vdots \\ 0\end{array}\right] U_{-N}^{n}+\left[\begin{array}{l}0 \\ 0 \\ \vdots \\ -\nu / 2\end{array}\right] U_{N}^{n}$.

For the Lax-Wendroff scheme the matrix $A$ is given by the matrix $A_{L W}$, where

$$
A_{L W}=\left[\begin{array}{llll}
1-\nu^{2} & \nu(\nu-1) / 2 & & \\
\nu(\nu+1) / 2 & & \ddots & \\
& \ddots & & \nu(\nu-1) / 2 \\
& & \nu(\nu+1) / 2 & 1-\nu^{2}
\end{array}\right]
$$


and the vector $\mathbf{v}_{A}^{n}$ is given by the vector $\mathbf{v}_{A_{L W}}^{n}$

$$
\mathbf{v}_{A_{L W}}^{n}=\left[\begin{array}{c}
\nu(\nu+1) / 2 \\
0 \\
\vdots \\
0
\end{array}\right] U_{-N}^{n}+\left[\begin{array}{c}
0 \\
0 \\
\vdots \\
\nu(\nu-1) / 2
\end{array}\right] U_{N}^{n} .
$$

\section{Convergence of the finite difference schemes}

In this section we study the convergence of the finite difference schemes. We start to prove the schemes are consistent with equation (1) and we determine under which conditions they are stable. The stability analysis is done by using two methods: the von Neumann analysis and the analysis of the behaviour of the norm of the matrix iteration of each scheme.

4.1. Consistency. In this section we analyse the truncation error, $T_{j}^{n}$, for the numerical schemes presented.

Let $u=u(x, t)$ be the solution of our equation. The fractional operator, $\nabla_{\beta}^{\alpha}$, in all the three schemes is approximated by $\delta_{\beta}^{\alpha} / 2 \Delta x^{\alpha}$ which is first order accurate as proved in [14], that is,

$$
\frac{\delta_{\beta}^{\alpha} u_{j}^{n}}{2 \Delta x^{\alpha}}=\left(\nabla_{\beta}^{\alpha} u\right)_{j}^{n}+\mathcal{O}(\Delta x)
$$

For the upwind scheme we have

$$
\begin{aligned}
T_{j}^{n} & =\frac{u_{j}^{n+1}-u_{j}^{n}}{\Delta t}+V \frac{u_{j}^{n}-u_{j-1}^{n}}{\Delta t}-\frac{D}{2 \Delta x^{\alpha}} \delta_{\beta}^{\alpha} u_{j}^{n} \\
& =\left(\frac{\partial u}{\partial t}\right)_{j}^{n}+\mathcal{O}(\Delta t)+V\left(\frac{\partial u}{\partial x}\right)_{j}^{n}+\mathcal{O}(\Delta x)-D\left(\nabla_{\beta}^{\alpha} u\right)_{j}^{n}+\mathcal{O}(\Delta x) .
\end{aligned}
$$

Therefore the upwind scheme has an order of accuracy $\mathcal{O}(\Delta t)+\mathcal{O}(\Delta x)+$ $\mathcal{O}(\Delta x)$. Similarly for the central scheme we have

$$
\begin{aligned}
T_{j}^{n} & =\frac{u_{j}^{n+1}-u_{j}^{n}}{\Delta t}+V \frac{u_{j+1}^{n}-u_{j-1}^{n}}{\Delta t}-\frac{D}{2 \Delta x^{\alpha}} \delta_{\beta}^{\alpha} u_{j}^{n} \\
& =\left(\frac{\partial u}{\partial t}\right)_{j}^{n}+\mathcal{O}(\Delta t)+V\left(\frac{\partial u}{\partial x}\right)_{j}^{n}+\mathcal{O}\left(\Delta x^{2}\right)-D\left(\nabla_{\beta}^{\alpha} u\right)_{j}^{n}+\mathcal{O}(\Delta x)
\end{aligned}
$$


and the order of accuracy is $\mathcal{O}(\Delta t)+\mathcal{O}\left(\Delta x^{2}\right)+\mathcal{O}(\Delta x)$. For the Lax-Wendroff scheme we have

$$
\begin{aligned}
T_{j}^{n}= & \frac{u_{j}^{n+1}-u_{j}^{n}}{\Delta t}+V \frac{u_{j+1}^{n}-u_{j-1}^{n}}{\Delta t}+V^{2} \frac{\Delta t}{2} \frac{u_{j+1}^{n}-2 u_{j}^{n}+u_{j-1}^{n}}{\Delta x^{2}}-\frac{D}{2 \Delta x^{\alpha}} \delta_{\beta}^{\alpha} u_{j}^{n} \\
= & \left(\frac{\partial u}{\partial t}\right)_{j}^{n}+\frac{\Delta t}{2}\left(\frac{\partial^{2} u}{\partial t^{2}}\right)_{j}^{n}+\mathcal{O}\left(\Delta t^{2}\right)+V\left(\frac{\partial u}{\partial x}\right)_{j}^{n}+\mathcal{O}\left(\Delta x^{2}\right) \\
& +V^{2} \frac{\Delta t}{2}\left(\frac{\partial^{2} u}{\partial x^{2}}\right)_{j}^{n}+\mathcal{O}\left(\Delta x^{2}\right)-D\left(\nabla_{\beta}^{\alpha} u\right)_{j}^{n}+\mathcal{O}(\Delta x) .
\end{aligned}
$$

The order of accuracy is $\mathcal{O}(\Delta t)+\mathcal{O}\left(\Delta x^{2}\right)+\mathcal{O}(\Delta x)$. For small $D$, from (15), it follows

$$
T_{j}^{n}=\left(\frac{\partial u}{\partial t}\right)_{j}^{n}+\mathcal{O}\left(\Delta t^{2}\right)+V\left(\frac{\partial u}{\partial x}\right)_{j}^{n}+\mathcal{O}\left(\Delta x^{2}\right)+\mathcal{O}\left(\Delta x^{2}\right)-D\left(\nabla_{\beta}^{\alpha} u\right)_{j}^{n}+\mathcal{O}(\Delta x),
$$

and the Lax-Wendroff scheme has an order of accuracy close to $\mathcal{O}\left(\Delta t^{2}\right)+$ $\mathcal{O}\left(\Delta x^{2}\right)+\mathcal{O}(\Delta x)$.

4.2. Stability analysis of the finite difference schemes. In order to derive stability conditions for the finite difference schemes, we apply the von Neumann analysis or Fourier analysis. Moreover, we show stability conditions obtained by computing numerically the norm of the matrix iteration of each scheme.

The von Neumann analysis assumes that any finite mesh function, such as, the numerical solution $U_{j}^{n}$ will be decomposed into a Fourier series as

$$
U_{j}^{n}=\sum_{p=-N}^{N} \kappa_{p}^{n} e^{i \xi_{p}(j \Delta x)}, \quad j=-N, \ldots, N,
$$

where $\kappa_{p}^{n}$ is the amplitude of the $p$-th harmonic and $\xi_{p}=p \pi / N \Delta x$. The product $\xi_{p} \Delta x$ is often called the phase angle $\theta=\xi_{p} \Delta x$ and covers the domain $[-\pi, \pi]$ in steps of $\pi / N$.

Considering a single mode $\kappa^{n} e^{i j \theta}$, its time evolution is determined by the same numerical scheme as the complete numerical solution $U_{j}^{n}$. Hence inserting a representation of this form into a numerical scheme we obtain stability conditions. The stability conditions will be satisfied if the amplitude factor $\kappa$ does not grow in time, that is, if we have $|\kappa(\theta)| \leq 1$, for all $\theta$. 
First we start to give some straightforward properties that are used to prove the stability results.

Proposition 1. The coefficients $g_{k}, k=0, \ldots, 2 N$ satisfy

(i) $g_{0}=1, \quad g_{1}=-\alpha, \quad g_{2}=\frac{\alpha(\alpha-1)}{2 !}$ and $\quad g_{k}>0, \quad k \geq 3$.

(ii) $\sum_{k=2}^{\infty} g_{k}=\alpha-1$.

Proof: (i) We can write $g_{k}$ as

$$
g_{0}=1 \quad \text { and } \quad g_{k+1}=-\frac{(\alpha-k)}{k+1} g_{k}, k \geq 1
$$

In particular, we have $g_{1}=-\alpha$ and $g_{2}=\alpha(\alpha-1) / 2>0$. Since $\alpha-k<0$ for $k \geq 3$, by induction we can conclude that $g_{k}>0$ for $k \geq 3$.

(ii) This result follows directly from $\sum_{k=0}^{\infty} g_{k}=0$, since $g_{0}+g_{1}+\sum_{k=2}^{\infty} g_{k}=0$ for $g_{0}=1$ and $g_{1}=-\alpha$.

Let us start to consider the discretisation of the fractional diffusion equation, that is, of the equation (1) with $V=0$. We have

$$
U_{j}^{n+1}=U_{j}^{n}+\frac{1}{2} \mu_{\alpha} \delta_{\beta}^{\alpha} U_{j}^{n},
$$

that is,

$$
U_{j}^{n+1}=U_{j}^{n}+\frac{1}{2} \mu_{\alpha}\left[(1+\beta) \sum_{k=0}^{N+j+1} g_{k} U_{j+1-k}+(1-\beta) \sum_{k=0}^{N-j+1} g_{k} U_{j-1+k}\right] .
$$

Note that for $\alpha=2$, we have $g_{0}=1, g_{1}=-2, g_{2}=1$ and $g_{k}=0$ for all $k \geq 3$.

The following theorem concerns the stability of the numerical scheme (21).

Theorem 2. Let $-1 \leq \beta \leq 1$ and $1<\alpha \leq 2$. If the numerical scheme (21) is von Neumann stable, then $\mu_{\alpha} \leq 2^{1-\alpha}$.

Proof: If we insert the mode $\kappa^{n} e^{i j \theta}$ into the scheme (21), we obtain the following amplification factor

$$
\kappa(\theta)=1+\frac{1}{2} \mu_{\alpha}\left\{(1+\beta) \sum_{k=0}^{N+j+1} g_{k} e^{i(1-k) \theta}+(1-\beta) \sum_{k=0}^{N-j+1} g_{k} e^{-i(1-k) \theta}\right\} .
$$


Let us consider $\theta=0$ and $\theta=\pi$. Note that the region around $\theta=0$ corresponds to the low frequencies while the region around $\theta=\pi$ is associated with the high-frequencies. In particular, the value $\theta=\pi$ corresponds to the highest frequency resolvable on the mesh, namely frequency of wavelength $2 \Delta x$.

For $\theta=0$ we have

$$
\begin{aligned}
\kappa(0) & =1+\frac{1}{2} \mu_{\alpha}\left\{(1+\beta) \sum_{k=0}^{N+j+1} g_{k}+(1-\beta) \sum_{k=0}^{N-j+1} g_{k}\right\} \\
& =1+\mu_{\alpha}\left(g_{0}+g_{1}\right)+\frac{1}{2} \mu_{\alpha}\left\{(1+\beta) \sum_{k=2}^{N+j+1} g_{k}+(1-\beta) \sum_{k=2}^{N-j+1} g_{k}\right\} \\
& \leq 1+\mu_{\alpha}(1-\alpha)+\frac{1}{2} \mu_{\alpha}[(1+\beta)(\alpha-1)+(1-\beta)(\alpha-1)]=1 .
\end{aligned}
$$

For $\theta=\pi$, the amplification factor is given by

$$
\begin{aligned}
\kappa(\pi)= & 1+\frac{1}{2} \mu_{\alpha}\left\{(1+\beta) \sum_{k=0}^{N+j+1} g_{k} \cos ((1-k) \pi)\right. \\
& \left.+(1-\beta) \sum_{k=0}^{N-j+1} g_{k} \cos ((1-k) \pi)\right\} .
\end{aligned}
$$

Since $\cos ((1-k) \pi)=(-1)^{k-1}$ it follows

$$
\begin{aligned}
\kappa(\pi) & =1+\frac{1}{2} \mu_{\alpha}\left\{-(1+\beta) \sum_{k=0}^{N+j+1} a_{k}-(1-\beta) \sum_{k=0}^{N-j+1} a_{k}\right\} \\
& =1-\frac{1}{2} \mu_{\alpha}\left\{(1+\beta) \sum_{k=0}^{N+j+1} a_{k}+(1-\beta) \sum_{k=0}^{N-j+1} a_{k}\right\},
\end{aligned}
$$

where $a_{k}=\left(\begin{array}{c}\alpha \\ k\end{array}\right)$. The condition $|\kappa(\pi)| \leq 1$ is equivalent to

$$
\frac{1}{2} \mu_{\alpha}\left\{(1+\beta) \sum_{k=0}^{N+j+1} a_{k}+(1-\beta) \sum_{k=0}^{N-j+1} a_{k}\right\} \leq 2 .
$$


Therefore, since $\sum_{k=0}^{\infty} a_{k}=2^{\alpha}$, we get

$$
\begin{aligned}
\frac{1}{2} \mu_{\alpha}\left\{(1+\beta) \sum_{k=0}^{N+j+1} a_{k}+(1-\beta) \sum_{k=0}^{N-j+1} a_{k}\right\} & \leq \frac{1}{2} \mu_{\alpha}\left[(1+\beta) 2^{\alpha}+(1-\beta) 2^{\alpha}\right] \\
& =\mu_{\alpha} 2^{\alpha} .
\end{aligned}
$$

Hence, we must have $\mu_{\alpha} 2^{\alpha} \leq 2$, that is, $\mu_{\alpha} \leq 2^{1-\alpha}$.

The following stability results are for the upwind and Lax-Wendroff schemes. The proofs are technically similar.

Theorem 3. Let $-1 \leq \beta \leq 1$ and $1<\alpha \leq 2$. If the upwind scheme (11) is von Neumann stable, then $\nu+2^{\alpha-1} \mu_{\alpha} \leq 1$.

Proof: For the upwind scheme the amplification factor is given by

$$
\begin{aligned}
\kappa_{U}(\theta)= & 1-\nu\left(1-e^{-i \theta}\right)+\frac{1}{2} \mu_{\alpha}\left\{(1+\beta) \sum_{k=0}^{N+j+1} g_{k} e^{i(1-k) \theta}\right. \\
& \left.+(1-\beta) \sum_{k=0}^{N-j+1} g_{k} e^{-i(1-k) \theta}\right\} .
\end{aligned}
$$

For $\theta=\pi$ it follows

$$
\kappa_{U}(\pi)=1-2 \nu-\frac{1}{2} \mu_{\alpha}\left\{(1+\beta) \sum_{k=0}^{N+j+1} a_{k}+(1-\beta) \sum_{k=0}^{N-j+1} a_{k}\right\} .
$$

Therefore to have $\left|\kappa_{U}(\pi)\right| \leq 1$ we must have

$$
2 \nu+\frac{1}{2} \mu_{\alpha}\left\{(1+\beta) \sum_{k=0}^{N+j+1} a_{k}+(1-\beta) \sum_{k=0}^{N-j+1} a_{k}\right\} \leq 2 .
$$

Moreover

$$
2 \nu+\frac{1}{2} \mu_{\alpha}\left\{(1+\beta) \sum_{k=0}^{N+j+1} a_{k}+(1-\beta) \sum_{k=0}^{N-j+1} a_{k}\right\} \leq 2 \nu+\mu_{\alpha} 2^{\alpha}
$$

and $2 \nu+\mu_{\alpha} 2^{\alpha} \leq 2$ implies $\nu+\mu_{\alpha} 2^{\alpha-1} \leq 1$.

Theorem 4. Let $-1 \leq \beta \leq 1$ and $1<\alpha \leq 2$. If the Lax-Wendroff scheme (17) is von Neumann stable, then $\nu^{2}+2^{\alpha-1} \mu_{\alpha} \leq 1$. 
Proof: The amplification factor for the Lax-Wendroff scheme is of the form

$$
\begin{aligned}
\kappa_{L W}(\theta)= & 1-\frac{\nu}{2}\left(e^{i \theta}-e^{-i \theta}\right)+\frac{\nu^{2}}{2}\left(e^{i \theta}-2+e^{-i \theta}\right) \\
& +\frac{1}{2} \mu_{\alpha}\left\{(1+\beta) \sum_{k=0}^{N+j+1} g_{k} e^{i(1-k) \theta}+(1-\beta) \sum_{k=0}^{N-j+1} g_{k} e^{-i(1-k) \theta}\right\} .
\end{aligned}
$$

For $\theta=\pi$ we have

$$
\kappa_{L W}(\pi)=1-2 \nu^{2}-\frac{1}{2} \mu_{\alpha}\left\{(1+\beta) \sum_{k=0}^{N+j+1} a_{k}+(1-\beta) \sum_{k=0}^{N-j+1} a_{k}\right\} .
$$

Similarly to have $\left|\kappa_{L W}(\pi)\right| \leq 1$ we must have

$$
2 \nu^{2}+\frac{1}{2} \mu_{\alpha}\left\{(1+\beta) \sum_{k=0}^{N+j+1} a_{k}+(1-\beta) \sum_{k=0}^{N-j+1} a_{k}\right\} \leq 2 .
$$

Moreover

$$
2 \nu^{2}+\frac{1}{2} \mu_{\alpha}\left\{(1+\beta) \sum_{k=0}^{N+j+1} a_{k}+(1-\beta) \sum_{k=0}^{N-j+1} a_{k}\right\} \leq 2 \nu^{2}+\mu_{\alpha} 2^{\alpha}
$$

and $2 \nu^{2}+\mu_{\alpha} 2^{\alpha} \leq 2$ implies $\nu^{2}+\mu_{\alpha} 2^{\alpha-1} \leq 1$.

The derivation of stability conditions for the central scheme is harder than for the previous schemes. For the central scheme of the classical advection diffusion equation, that is, for $\alpha=2$, it was also hard. In fact, the stability analysis for this scheme was controversial for some years because of the apparent difficulty of obtaining stability conditions, see for instance [20]. The following theorem concerning the central scheme sets out necessary stability conditions. These conditions are not as strong as the necessary conditions of the previous theorems, in the sense that they are less close to the respective necessary and sufficient stability conditions, as we will see later on.

Theorem 5. Let $-1 \leq \beta \leq 1$ and $1<\alpha \leq 2$. If the central scheme (12) is von Neumann stable, then $2^{\alpha-1} \mu_{\alpha} \leq 1$.

Proof: For the central scheme the amplification factor is given by

$$
\kappa_{C}(\theta)=1-\frac{\nu}{2}\left(e^{i \theta}-e^{-i \theta}\right)
$$




$$
+\frac{1}{2} \mu_{\alpha}\left\{(1+\beta) \sum_{k=0}^{N+j+1} g_{k} e^{i(1-k) \theta}+(1-\beta) \sum_{k=0}^{N-j+1} g_{k} e^{-i(1-k) \theta}\right\} .
$$

For $\theta=\pi$ we have

$$
\kappa_{C}(\pi)=1-\frac{1}{2} \mu_{\alpha}\left\{(1+\beta) \sum_{k=0}^{N+j+1} a_{k}+(1-\beta) \sum_{k=0}^{N-j+1} a_{k}\right\}
$$

and from Theorem 2 we know that $\left|\kappa_{C}(\pi)\right| \leq 1$ implies $\mu_{\alpha} \leq 2^{1-\alpha}$.

The well-known similar results for the advection diffusion equation are the following.

Theorem 6. Let $\alpha=2$.

(i) The upwind scheme is von Neumann stable if, and only if, $\nu+2 \mu_{\alpha} \leq 1$.

(ii) The Lax-Wendroff scheme is von Neumann stable if, and only if, $\nu^{2}+$ $2 \mu_{\alpha} \leq 1$.

(iii) The central scheme is von Neumann stable if, and only if, $\nu^{2} \leq 2 \mu_{\alpha} \leq$ 1.

Proof: The proof of (i), (ii) and (iii) can be found in various works such as [21], [22] and [20] respectively.

We have derived stability conditions for our schemes by using the von Neumann analysis. In what follows, we shall present stability conditions by computing numerically the 2-norm of the matrix iteration of each scheme.

Let us consider the matricial form of the numerical schemes discussed in section 4. For the exact solution, we denote $\mathbf{u}^{n}$ the set $\mathbf{u}^{n}:=\left\{u\left(x_{j}, t_{n}\right)\right\}$. Any errors in a calculation based on (18) will grow according to

$$
E^{n+1}=M E^{n}+\Delta t T^{n}, \quad n=0,1,2, \ldots
$$

where $E^{n}$ is the error $E^{n}=\mathbf{u}^{n}-\mathbf{U}^{n}$ for the set of nodal errors and $T^{n}$ is the truncation error analised previously. For any chosen norm for the error, a practical stability requirement is the condition

$$
\|M\| \leq 1 \text {. }
$$

Therefore a global error bound is given by

$$
\left\|E^{n}\right\| \leq\left\|E^{0}\right\|+\Delta t \sum_{j=0}^{n-1}\left\|T^{j}\right\| \leq\left\|E^{0}\right\|+(n \Delta t) \max _{0 \leq j \leq n-1}\left\|T^{j}\right\| .
$$


Although there are cases for which the condition (23) is too restrictive, in some situations, such as, problems with periodic boundary conditions and Dirichlet boundary conditions, the stability conditions derived by using condition (23) with the 2-norm, are equivalent to the stability conditions obtained with von Neumann analysis [23]. The conditions derived here, using the property (23), allow to conclude that some of the analytical necessary conditions obtained with the von Neumann analysis are necessary and sufficient conditions for stability.

In Figures 1 to 6 we plot $\|M\| \leq 1$, where $\|\cdot\|$ is the 2 -norm, for different values of $\beta$ and $\alpha$.

Figure 1 shows the stability regions for the upwind and Lax-Wendroff schemes when $\beta=0$. The results displayed were obtained with condition (23) and they are the same as the stability regions defined by the conditions given in Theorem 3 and Theorem 4 respectively. In Figure 2 we observe the regions given by property (23) verify more conditions additionally to the necessary stability condition presented in Theorem 5. In Figures $1-2$ the regions for larger $\alpha$ are smaller.
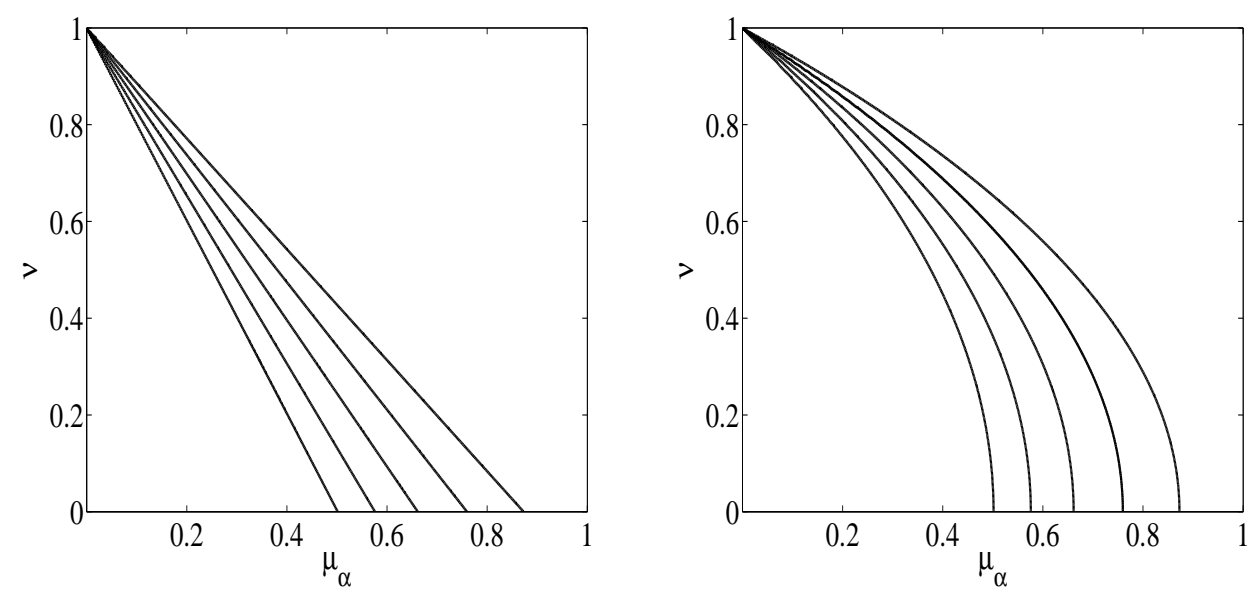

FIGURE 1. Stability regions obtained with condition (23) for $\beta=0$ and $\alpha=2,1.8,1.6,1.4,1.2$ (left to right). (a) Upwind scheme (11): these regions are defined by $\nu+2^{\alpha-1} \mu_{\alpha} \leq 1$ also given in Theorem 3; For different values of $\beta$ the stability regions are the same. (b) Lax-Wendroff scheme (17): these regions are defined by $\nu^{2}+2^{\alpha-1} \mu_{\alpha} \leq 1$ also given in Theorem 4 . For values of $\beta$ such as $-0.5 \leq \beta \leq 1$ the stability regions are the same.

In the case of the upwind for any $\beta$, we obtain the same stability conditions as for $\beta=0$. 


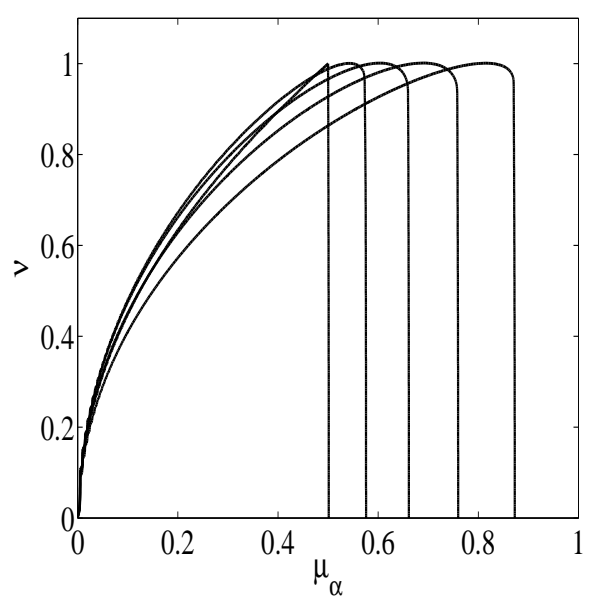

FIGURE 2. Stability regions obtained with condition (23) for the central scheme (12), with $\beta=0$ and $\alpha=2,1.8,1.6,1.4,1.2$ (left to right). One of the conditions that helps to define these regions is $2^{\alpha-1} \mu_{\alpha} \leq 1$ given in Theorem 5 .
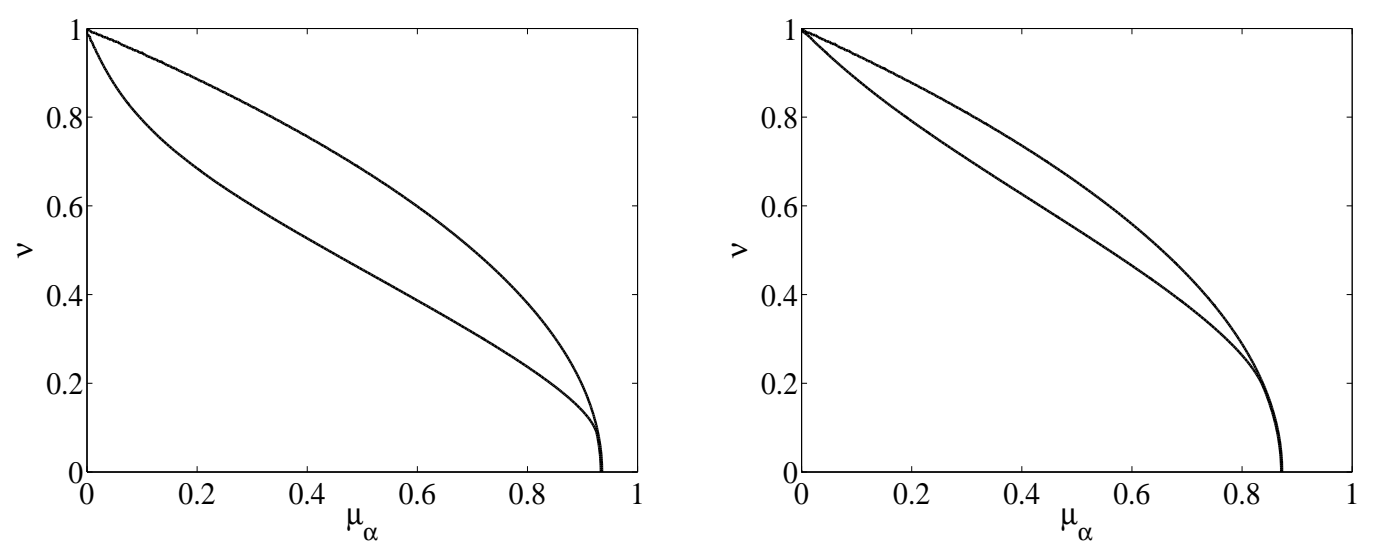

FIgURE 3. Stability regions obtained with condition (23) for the LaxWendroff scheme (12): (a) $\alpha=1.1$ - The curves are plotted for $\beta=-1,-0.6$ (left to right). For $-0.6 \leq \beta \leq 1$ the stability regions are the same as for $\beta=-0.6$; (b) $\alpha=1.2-$ The curves are plotted for $\beta=-1,-0.7$ (left to right). For $-0.7 \leq \beta \leq 1$ the stability regions are the same as for $\beta=-0.7$.

For the Lax-Wendroff scheme when $3 / 2 \leq \alpha \leq 2$ the stability regions for all $\beta$ are the same as for $\beta=0$, given in Theorem 4 as $\nu^{2}+2^{\alpha-1} \mu_{\alpha} \leq 1$ and shown in Figure 1.(b).

When $1<\alpha<3 / 2$ we have conditions slightly different for some negative values of $\beta$. For positive values of $\beta$ they are still the same as $\beta=0$. In Figures $3-4$ we plot the stability conditions for the Lax-Wendroff scheme 

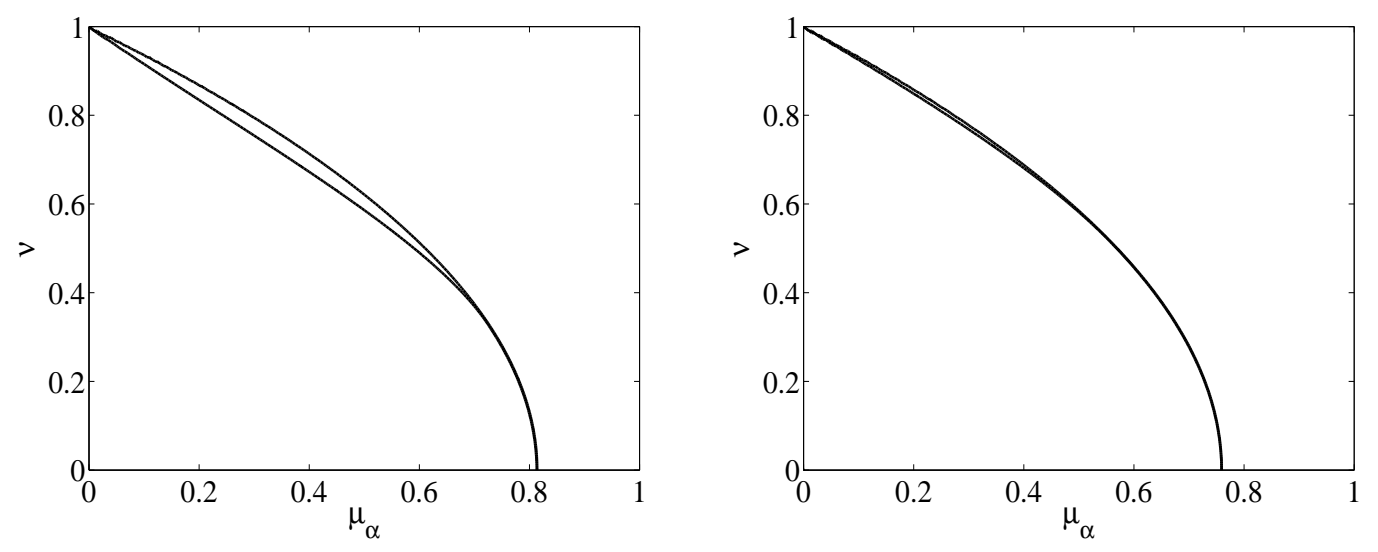

FIGURE 4. Stability regions obtained with condition (23) for the LaxWendroff scheme (12): (a) $\alpha=1.3$ - The curves are plotted for $\beta=-1,-0.8$ (left to right). For $-0.8 \leq \beta \leq 1$ the stability regions are the same as for $\beta=-0.8$; (b) $\alpha=1.4$ - The curves are plotted for $\beta=-1,-0.9$ (left to right). For $-0.9 \leq \beta \leq 1$ the stability regions are the same as for $\beta=-0.9$.

when $\alpha=1.1,1.2,1.3,1.4$ to see the effect of changing $\beta$ on the stability of the scheme.

For $\alpha=1.1$, when $-0.6 \leq \beta \leq 1$ the stability regions are the same as for $\beta=-0.6$, plotted in Figure 3.(a). In this figure we plot the stability curve for $\beta=-1$ and $\beta=-0.6$. The curves that define the stability conditions for $-1<\beta<-0.6$ fit between the two curves plotted. Moreover, for $\beta=-1$ the stability regions are smaller than for the rest of the $\beta$ values, that is, as $\beta$ increases the regions become bigger. A similar behaviour is observed in the examples that follow.

For $\alpha=1.2$ and $-0.7 \leq \beta \leq 1$ the stability regions are the same as for $\beta=-0.7$, shown in Figure 3.(b). Similarly, for $\alpha=1.3$ and $-0.8 \leq \beta \leq 1$ the stability regions are the same as for $\beta=-0.8$, plotted in Figure 4.(a). Finally, for $\alpha=1.4$, when $-0.9 \leq \beta \leq 1$, the stability regions are the same as for $\beta=-0.9$. The curves for $\beta=-1$ and $\beta=-0.9$ are mainly the same as can be seen in Figure 4.(b).

For the central scheme we obtain different regions for different values of $\beta$ and $\alpha$ as can be seen in Figures $5-6$. As for the previous cases the stability regions are bigger for larger values of $\beta$, that is, for $\beta=-1$ we have the smallest region and for $\beta=1$ the biggest region. For this scheme, according to Theorem 5 , we know that $2^{\alpha-1} \mu_{\alpha} \leq 1$. This condition is clearly displayed in Figures $5-6$, additionally to other conditions that we were unable to determine analytically. 

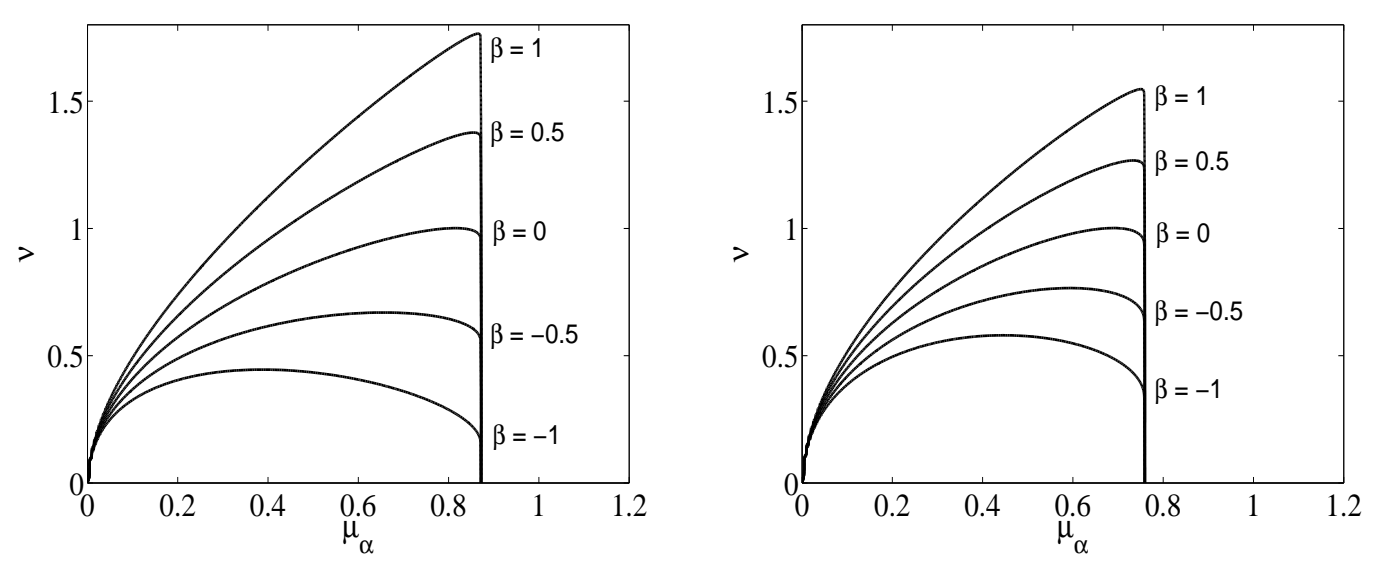

FIGURE 5. Stability regions obtained with condition (23) for the central scheme (17), with $\beta=-1,-0.5,0,0.5,1$ (bottom to top): (a) $\alpha=1.2$; (b) $\alpha=1.4$.
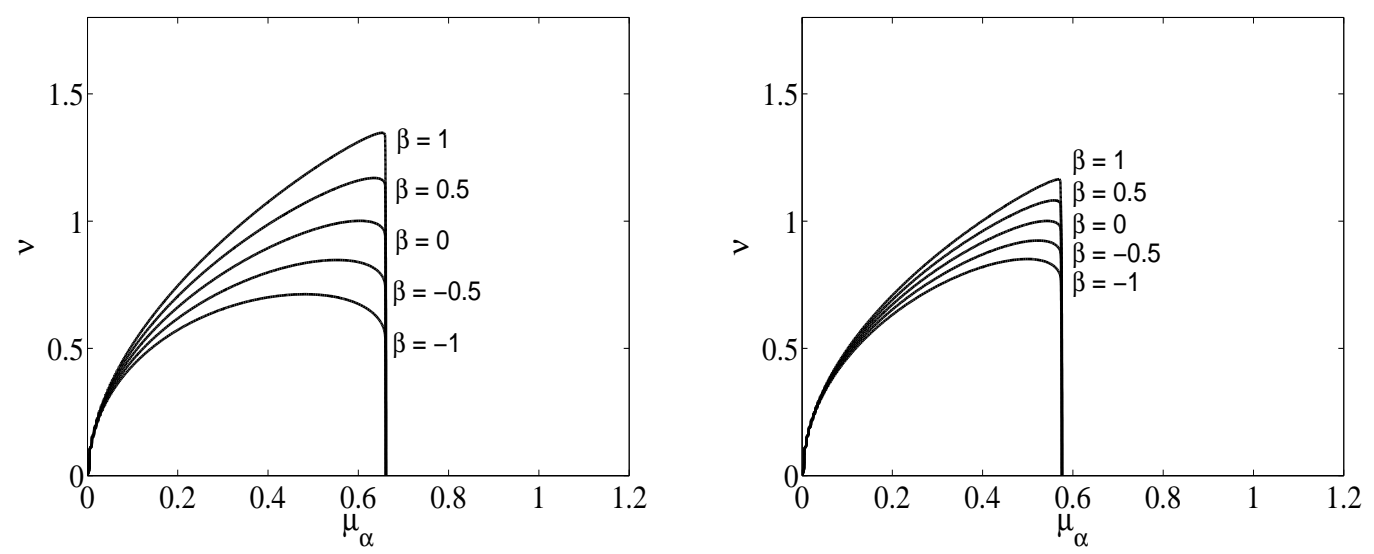

FIGURE 6. Stability regions obtained with condition (23) for the central scheme (17), with $\beta=-1,-0.5,0,0.5,1$ (bottom to top): (a) $\alpha=1.6$; (b) $\alpha=1.8$.

In general and for all the three schemes, for smaller $\alpha$ the stability regions are bigger, see Figure $1-6$. In practical computations this represents an advantage. Additionally, as $\Delta x \rightarrow 0, \Delta x^{\alpha}$ is larger for smaller $\alpha$ 's and $\mu_{\alpha}$ is smaller for smaller $\alpha$ 's and therefore it is easier to be inside the stability region when running the numerical tests.

We close this section, by remarking that the stability region of the LaxWendroff scheme presents more advantages than the stability region of the central scheme, since it is less restrictive. More in particular, for the central scheme, when $\mu_{\alpha}$ is small, the Courant number $\nu$ needs to be small for all $\alpha$ and $\beta$. 


\section{Numerical example}

In this section we start to derive an exact solution for a fractional advection diffusion problem. We include the details on how to obtain the exact solution since we belief this is of interest to the reader. Next, we present the numerical solution of the problem by running experiments with the three numerical methods discussed previously and the errors are measured by comparing the numerical solution with the exact solution.

5.1. Exact solution. Let us consider the problem when the transition of the solute particle is symmetric, that is, $\beta=0$. We have

$$
\frac{\partial u}{\partial t}+V \frac{\partial u}{\partial x}=D \nabla^{\alpha} u
$$

for

$$
2 \nabla^{\alpha} u=\frac{\partial^{\alpha} u}{\partial x^{\alpha}}+\frac{\partial^{\alpha} u}{\partial(-x)^{\alpha}}
$$

We define a problem on the whole line, $x \in \mathbb{R}$, and $t>0$. The initial condition is defined as

$$
u(x, 0)= \begin{cases}u_{0}, & x \leq 0 \\ 0, & x>0\end{cases}
$$

where $u_{0}$ is a constant. The boundary conditions are given by

$$
\lim _{x \rightarrow-\infty} u(x, t)=u_{0} \quad \text { and } \quad \lim _{x \rightarrow \infty} u(x, t)=0 .
$$

In the following proposition, we derive the exact solution by using Fourier transforms.

Proposition 7. The exact solution for the fractional advection diffusion equation (24) subject to the initial condition (25) and with boundary conditions (26) is of the form

$$
u(x, t)=u_{0}\left[1-F_{\alpha}\left(\frac{x-V t}{(R D t)^{1 / \alpha}}\right)\right]
$$

where $F_{\alpha}$ is the cumulative probability function and $R=\left|\cos \left(\frac{\pi \alpha}{2}\right)\right|$. For $\alpha \neq$ 1 and $x \geq 0$ the cumulative probability function is defined by

$$
F_{\alpha}(x)=1-\frac{1}{2} \int_{0}^{1} \exp \left[-x^{\alpha /(\alpha-1)} U_{\alpha}(\phi)\right] d \phi,
$$


where

$$
U_{\alpha}(\phi)=\left[\frac{\sin (\pi \alpha \phi / 2)}{\cos (\pi \phi / 2)}\right]^{\frac{\alpha}{1-\alpha}} \frac{\sin (\pi(\alpha-1) \phi / 2)}{\cos (\pi \phi / 2)} .
$$

The function $F_{\alpha}(x)$ for $\alpha \neq 0$ and $x<0$ is computed using the identity

$$
F_{\alpha}(-x)=1-F_{\alpha}(x) .
$$

Note that $F_{\alpha}(-\infty)=0$ and $F_{\alpha}(\infty)=1$.

Proof: Applying the Fourier transform at (24) we obtain

$$
\frac{d}{d t} \hat{u}(k, t)=i k V \hat{u}(k, t)+\frac{1}{2} D(-i k)^{\alpha} \hat{u}(k, t)+\frac{1}{2} D(i k)^{\alpha} \hat{u}(k, t)
$$

where the well-known Fourier transforms for integer derivatives

$$
\mathcal{F}\left[\left(d^{n} / d x^{n}\right) f(x)\right]=(i k)^{n} f(x)
$$

are extended to rational order

$$
\mathcal{F}\left[\frac{\partial^{\alpha} u}{\partial(x)^{\alpha}}\right]=(i k)^{\alpha} \hat{f}(k), \quad \mathcal{F}\left[\frac{\partial^{\alpha} u}{\partial(-x)^{\alpha}}\right]=(-i k)^{\alpha} \hat{f}(k) .
$$

This is an ordinary differential equation which solution is given by

$$
\hat{u}(k, t)=A \exp \left(\frac{1}{2}(-i k)^{\alpha} D t+\frac{1}{2}(i k)^{\alpha} D t+i k V t\right),
$$

where the constant $A$ is determined using the initial condition, that is, $A=$ $\hat{u}(k, 0)$. After some algebra we can write

$$
\hat{u}(k, t)=\hat{u}(k, 0) \exp \left(|\cos (\pi \alpha / 2)| D t|k|^{\alpha}+i k V t\right) .
$$

Therefore

$$
\hat{u}(k, t)=\hat{u}(k, 0) \psi(k),
$$

where

$$
\psi(k)=\exp \left(|\cos (\pi \alpha / 2)| D t|k|^{\alpha}+i k V t\right) .
$$

We note that $\psi(k)$ is a characteristic function. The cumulative probability function determined by the characteristic function and the density which is the differentiation of the cumulative probability will be denoted by $F_{\alpha}(x)$ and $f_{\alpha}(x)$ respectively. They are given by McCulloh et al [24], where

$$
\mathcal{F}^{-1}[\psi(k)]=f_{\alpha}\left(\frac{x-V t}{(R D t)^{1 / \alpha}}\right)
$$


and $F_{\alpha}^{\prime}(x)=f_{\alpha}(x)$, for $F_{\alpha}(x)$ defined by (28). Consequently, using the convolution property for Fourier transforms the inversion of (32) is given by

$$
u(x, t)=\int_{-\infty}^{\infty} u(\tau, 0) f_{\alpha}(y-\tau) d \tau
$$

where

$$
y=\frac{x-V t}{(R D t)^{1 / \alpha}} \quad \text { and } \quad R=\left|\cos \left(\frac{\pi \alpha}{2}\right)\right| .
$$

Since $u(x, 0)=0$ for $x>0$ and $u(x, 0)=u_{0}$ for $x \leq 0$ we have

$$
u(x, t)=\int_{-\infty}^{0} u_{0} f_{\alpha}(y-\tau) d \tau=u_{0} \int_{y}^{\infty} f_{\alpha}(\xi) d \xi .
$$

Therefore

$$
u(x, t)=u_{0}\left[\lim _{\xi \rightarrow \infty} F_{\alpha}(\xi)-F_{\alpha}(y)\right]=u_{0}\left[1-F_{\alpha}(y)\right]
$$

Finally,

$$
u(x, t)=u_{0}\left[1-F_{\alpha}\left(\frac{x-V t}{(R D t)^{1 / \alpha}}\right)\right] .
$$

Consider now the definition of a $\alpha$-stable error function, $\operatorname{Serf}_{\alpha}$

$$
\operatorname{Serf}_{\alpha}(z)=2 \int_{0}^{z} f_{\alpha}(x) d x
$$

Note that

$$
\operatorname{Serf}_{\alpha}(z)=2 \int_{0}^{z} f_{\alpha}(x) d x=2\left(\int_{-\infty}^{z} f_{\alpha}(x)-\frac{1}{2}\right)
$$

Therefore we can also write the solution (27) in the form

$$
u(x, t)=\frac{u_{0}}{2}\left[1-\operatorname{Serf}_{\alpha}\left(\frac{x-V t}{(R D t)^{1 / \alpha}}\right)\right] .
$$

For $\alpha=2$, which is the case equation (24) turns to be the conventional advection-diffusion equation, the solution is given by

$$
u(x, t)=\frac{u_{0}}{2}\left[1-\operatorname{Erf}\left(\frac{x-V t}{2 \sqrt{D t}}\right)\right],
$$

where Erf is the error function. 
A similar solution to this one is the named Ogata and Banks solution [25]

$$
u(x, t)=\frac{u_{0}}{2}\left[1-\operatorname{Erf}\left(\frac{x-V t}{2 \sqrt{D t}}\right)+e^{V x / D} \operatorname{Erfc}\left(\frac{x+V t}{2 \sqrt{D t}}\right)\right],
$$

where Erfc is the complementary error function. This is a solution of a slightly different problem which is defined in half-line, that is, $x \geq 0$ with initial condition

$$
u(x, 0)=0
$$

and boundary conditions

$$
u(0, t)=u_{0} \quad \text { and } \quad u(\infty, t)=0 .
$$

We note that for very small diffusion the solutions are basically the same. Ogata and Banks [25] show that when the Peclet number $V x / D$ is less than 500 we can neglect the second term of (36) and therefore we obtain the same solution as (35).

5.2. Test problem. Consider the fractional differential equation (24), for $x$ defined in the interval $[-L, L], t>0$ and subject to the initial condition

$$
u(x, 0)= \begin{cases}1, & x \leq 0 \\ 0, & x>0\end{cases}
$$

We consider $L$ sufficiently large such that the boundary conditions are given by

$$
u(-L, t)=1 \quad \text { and } \quad u(L, t)=0 .
$$

In Figure 7, we plot the exact solution (27) for $V=0.5, D=0.2$ at $t=1$. We consider $\alpha=1.8,1.6,1.4,1.2$.

Consider the vector $u_{e x}=\left(u\left(x_{-N}, t\right), \ldots, u\left(x_{0}, t\right), \ldots, u\left(x_{N}, t\right)\right)$, where $u$ is the exact solution $(27)$ and $U_{a p p}=\left(U\left(x_{-N}, t\right), \ldots, U\left(x_{0}, t\right), \ldots, U\left(x_{N}, t\right)\right)$, where $U$ is the approximated solution given by the respective numerical method. The error is defined by

$$
\operatorname{Error}(\Delta x)=\left\|u_{e x}(\Delta x)-U_{a p p}(\Delta x)\right\|_{\infty},
$$

where $\|\cdot\|_{\infty}$ is the $L_{\infty}$ norm.

We display in Table 1 to Table 5 the global error results for $V=0.5$ and $D=0.2$ at $t=1$. In Table 1 we show the results for $\alpha=2$. For $\alpha=$ 2 , equation (1) becomes the advection-diffusion equation and the schemes upwind, central and Lax-Wendroff are well know schemes in the literature $[26],[21],[27]$. 


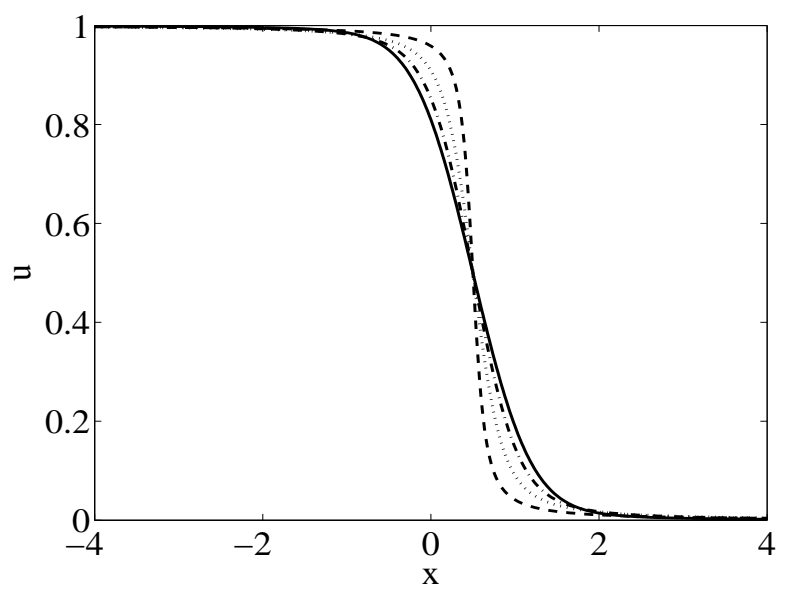

FiguRE 7. Exact solution (27) for $V=0.5, D=0.2$ at $t=1: \alpha=1.8(-)$; $\alpha=1.6(-\cdot-) ; \alpha=1.4(\cdots) ; \alpha=1.2(--)$.

From Table 1 to Table 5, we observe that the rate of convergence is around one for the three schemes and for $1<\alpha \leq 2$, as should be expected according to the analysis of section 4.1 .

As $\alpha$ becomes closer to one the rate of convergence is slightly smaller and the error is larger, see Table 5. This is related with the behaviour of the solution for smaller $\alpha$, since for smaller $\alpha$ the solution is less smoother, as can be seen in Figure 7, what makes the exact solution harder to approximate accurately.

The error of the central and Lax-Wendroff schemes is smaller than the error of the upwind scheme. This is illustrated clearly, for instance, in Table 3. Since we saw the Lax-Wendroff scheme has better stability conditions than the central scheme, we can infer the Lax-Wendroff scheme in general presents more advantages than the other two schemes.

\begin{tabular}{lllll}
\hline Schemes & $\Delta x=0.2$ & $\Delta x=0.02$ & $\Delta x=0.002$ & $\begin{array}{l}\text { Convergence } \\
\text { rate }\end{array}$ \\
\hline Upwind & $0.6671 \times 10^{-1}$ & $0.7662 \times 10^{-2}$ & $0.7772 \times 10^{-3}$ & 0.97 \\
Lax-Wendroff & $0.5848 \times 10^{-1}$ & $0.6262 \times 10^{-2}$ & $0.6617 \times 10^{-3}$ & 0.97 \\
Central & $0.5849 \times 10^{-1}$ & $0.6262 \times 10^{-2}$ & $0.6617 \times 10^{-3}$ & 0.97 \\
\hline
\end{tabular}

TABLE 1. Global $L_{\infty}$ error (38) of time converged solution for three mesh resolutions at $t=1$ for $\alpha=2$.

To conclude this section we display a number of figures, Figures $8-10$, to give an idea of the effect of parameters $\alpha$ and $\beta$. Note that when $\alpha=2$ 


\begin{tabular}{lllll}
\hline Schemes & $\Delta x=0.2$ & $\Delta x=0.02$ & $\Delta x=0.002$ & $\begin{array}{l}\text { Convergence } \\
\text { rate }\end{array}$ \\
\hline Upwind & $0.7675 \times 10^{-1}$ & $0.9193 \times 10^{-2}$ & $0.8666 \times 10^{-3}$ & 0.97 \\
Lax-Wendroff & $0.6289 \times 10^{-1}$ & $0.6989 \times 10^{-2}$ & $0.6930 \times 10^{-3}$ & 0.98 \\
Central & $0.6297 \times 10^{-1}$ & $0.6988 \times 10^{-2}$ & $0.6931 \times 10^{-3}$ & 0.98 \\
\hline
\end{tabular}

TABLE 2. Global $L_{\infty}$ error (38) of time converged solution for three mesh resolutions at $t=1$ for $\alpha=1.8$.

\begin{tabular}{lllll}
\hline Schemes & $\Delta x=0.2$ & $\Delta x=0.02$ & $\Delta x=0.002$ & $\begin{array}{l}\text { Convergence } \\
\text { rate }\end{array}$ \\
\hline Upwind & $0.1016 \times 10^{0}$ & $0.1340 \times 10^{-1}$ & $0.1191 \times 10^{-2}$ & 0.97 \\
Lax-Wendroff & $0.7156 \times 10^{-1}$ & $0.8808 \times 10^{-2}$ & $0.7364 \times 10^{-3}$ & 0.99 \\
Central & $0.7150 \times 10^{-1}$ & $0.8798 \times 10^{-2}$ & $0.7365 \times 10^{-3}$ & 0.99 \\
\hline
\end{tabular}

TABLE 3. Global $L_{\infty}$ error (38) of time converged solution for three mesh resolutions at $t=1$ for $\alpha=1.6$.

\begin{tabular}{lllll}
\hline Schemes & $\Delta x=0.2$ & $\Delta x=0.02$ & $\Delta x=0.002$ & $\begin{array}{l}\text { Convergence } \\
\text { rate }\end{array}$ \\
\hline Upwind & $0.1553 \times 10^{0}$ & $0.2684 \times 10^{-1}$ & $0.2632 \times 10^{-2}$ & 0.89 \\
Lax-Wendroff & $0.1005 \times 10^{0}$ & $0.1531 \times 10^{-1}$ & $0.1267 \times 10^{-2}$ & 0.95 \\
Central & $0.1002 \times 10^{0}$ & $0.1527 \times 10^{-1}$ & $0.1262 \times 10^{-2}$ & 0.95 \\
\hline
\end{tabular}

TABLE 4. Global $L_{\infty}$ error (38) of time converged solution for three mesh resolutions at $t=1$ for $\alpha=1.4$.

\begin{tabular}{lllll}
\hline Schemes & $\Delta x=0.2$ & $\Delta x=0.02$ & $\Delta x=0.002$ & $\begin{array}{l}\text { Convergence } \\
\text { rate }\end{array}$ \\
\hline Upwind & $0.2364 \times 10^{0}$ & $0.8397 \times 10^{-1}$ & $0.1321 \times 10^{-1}$ & 0.63 \\
Lax-Wendroff & $0.1935 \times 10^{0}$ & $0.4868 \times 10^{-1}$ & $0.5992 \times 10^{-2}$ & 0.75 \\
Central & $0.1931 \times 10^{0}$ & $0.4850 \times 10^{-1}$ & $0.5962 \times 10^{-2}$ & 0.76 \\
\hline
\end{tabular}

TABLE 5. Global $L_{\infty}$ error (38) of time converged solution for three mesh resolutions at $t=1$ for $\alpha=1.2$.

the equation (1) for $\beta=-1$ is the same as for $\beta=1$. The approximated solutions displayed in Figures 8 - 10 were obtained by running the LaxWendroff scheme. 

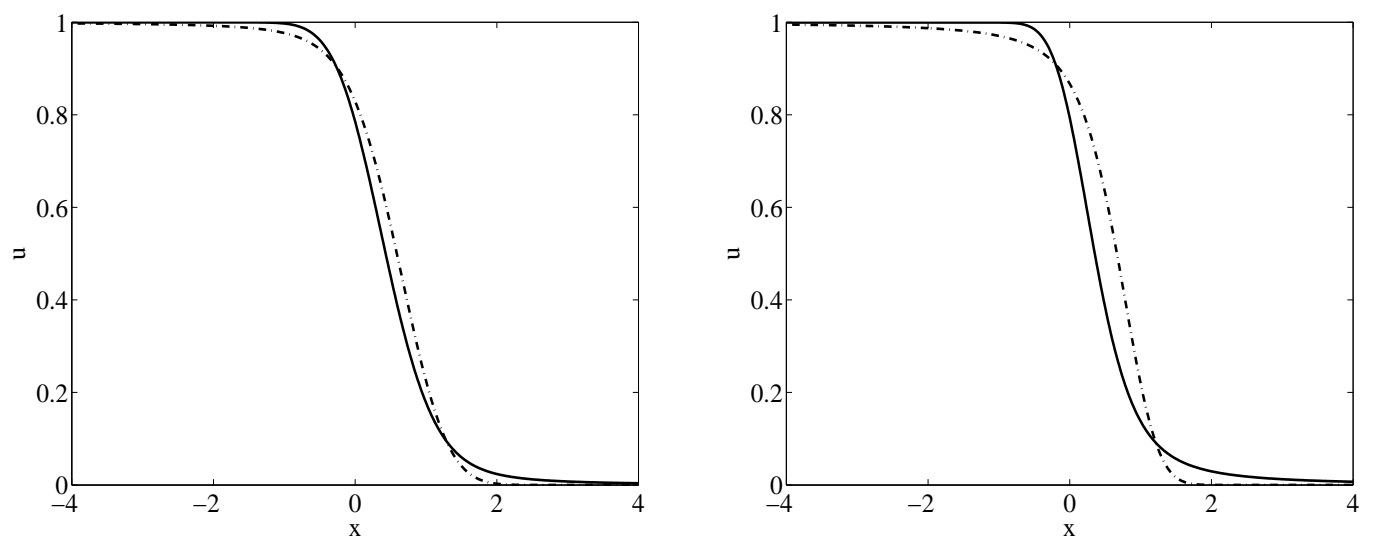

FiguRE 8. Solution of (1) for $V=0.5, D=0.2$ at $t=1$ and for $\beta=$ $-1(-\cdot-), \beta=1(-)$ : (a) $\alpha=1.8$; (b) $\alpha=1.6$.
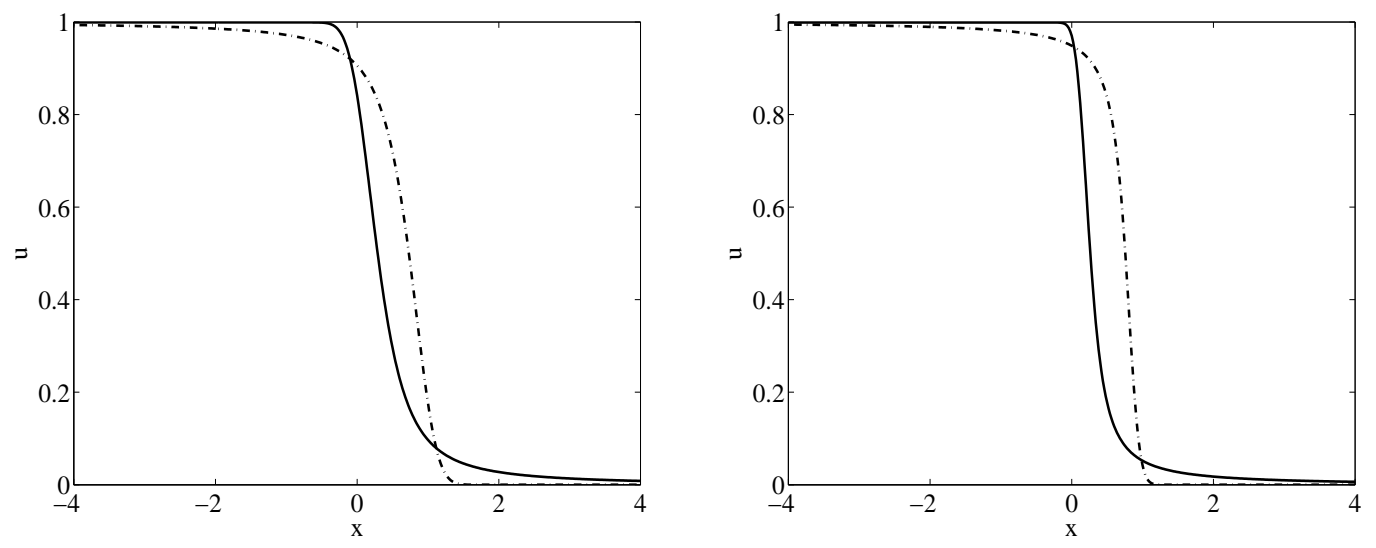

Figure 9. Solution of (1) for $V=0.5, D=0.2$ at $t=1$ and for $\beta=$ $-1(-\cdot-), \beta=1$ (-): (a) $\alpha=1.4$; (b) $\alpha=1.2$.
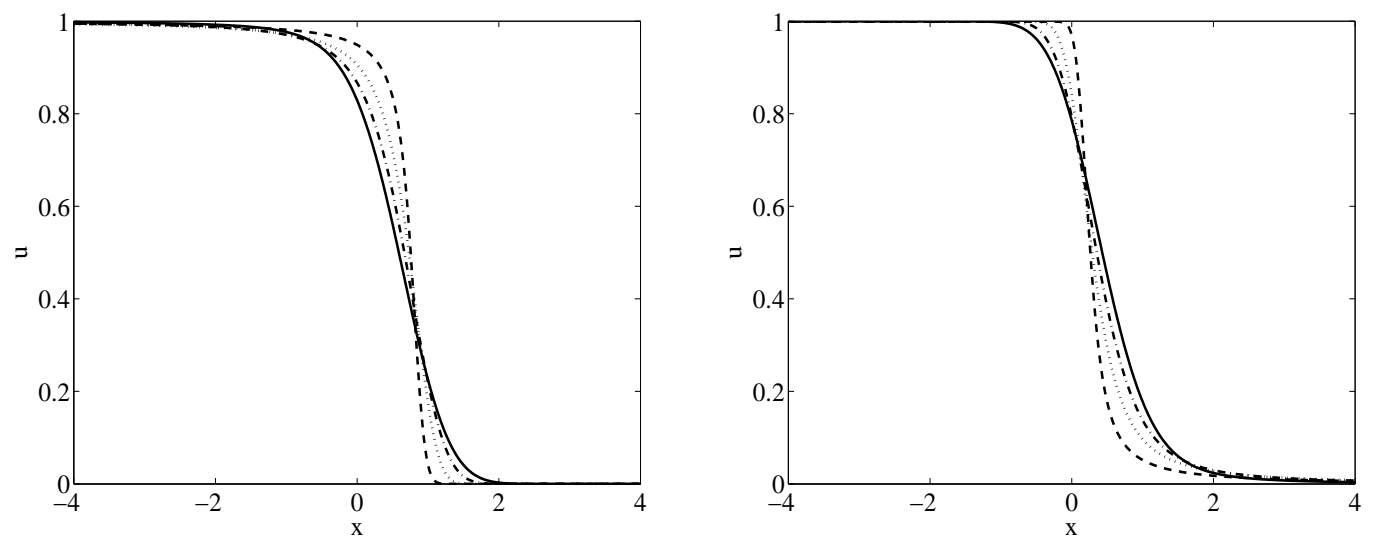

Figure 10. Solution of (1) for $V=0.5, D=0.2$ at $t=1$ and for $\alpha=1.8$ ($-) ; \alpha=1.6(-\cdot-)$; $\alpha=1.4(\cdots)$; $\alpha=1.2(--)$ : (a) $\beta=-1$; (b) $\beta=1$. 
E. SOUSA

\section{Conclusion}

Finite difference methods for solving fractional advection-diffusion problems are given and named respectively as upwind, central and Lax-Wendroff schemes. These numerical schemes can be seen as generalisations of existing schemes for the advection diffusion equation. The numerical methods are proved to be consistent and the order of convergence is one. Since they are consistent its convergence is proved by showing they are stable under certain conditions. The central and Lax-Wendroff schemes present smaller errors than the upwind scheme, although the rate of convergence is the same. Additionally the stability region of the Lax-Wendroff scheme is more adequate than the stability region of the central scheme since for small diffusive parameters $\mu_{\alpha}$ and larger Courant numbers $\nu$, the latter can be unstable.

\section{References}

[1] D.A. Benson, S.W. Wheatcraft, M.M. Meerschaert, Application of a fractional advectiondispersion equation, Water Resources Research 36 (2000) 1403-1412.

[2] G. Huang, Q. Huang, H. Zhan, Evidence of one-dimensional scale-dependent fractional advection-dispersion, Journal of Contaminant Hydrology 85 (2006) 53-71.

[3] R. Metzler, J. Klafter, The random walk's guide to anomalous diffusion: a fractional dynamics approach, Phys. Rep. 339 (2000) 1-77.

[4] R. Metzler, J. Klafter, Accelerating Brownian motion: a fractional dynamics approach to fast diffusion, Europhys. Lett. 51 (2000) 492-498.

[5] Y. Pachepsky, D. Benson, W. Rawls, Simulating scale-dependent solute transport in soils with the fractional advective-dispersive equation, Soil Sci. Soc. Am. J. 4 (2000) 1234-1243.

[6] L. Zhou, H.M. Selim, Application of the fractional advection-dispersion equation in porous media, Soil Sci. Soc. Am. J. 67 (2003) 1079-1084.

[7] C-M Chen, F. Liu, I. Turner, V. Anh, A Fourier method for the fractional diffusion equation describing sub-diffusion, Journal of Computational Physics 227 (2007) 886-897.

[8] S. Shen, F. Liu, Error analysis of an explicit finite difference approximation for the space fractional diffusion equation with insulated ends, ANZIAM J. 46 (E) (2005) C871-C887.

[9] C. Tadjeran, M.M. Meerschaert, H-P Scheffler, A second-order accurate numerical approximation for the fractional diffusion equation, Journal of Computational Physics 213 (2006) 205-213.

[10] C. Tadjeran, M.M. Meerschaert, A second-order accurate numerical approximation for the two-dimensional fractional diffusion equation, Journal of Computational Physics 220 (2007) $813-823$.

[11] S.B. Yuste, L. Acedo, An explicit finite difference method and a new von Neumann type stability analysis for fractional diffusion equations, SIAM J. Numer. Anal. 42 (2005) 18621874.

[12] S.B. Yuste, Weighted average finite difference methods for fractional diffusion equations, Journal of Computational Physics 216 (2006) 264-274.

[13] F. Liu, P. Zhuang, V. Anh, I. Turner, K. Burrage, Stability and convergence of the difference methods for the space-time fractional advection-diffusion equation, Applied Mathematics and Computation 191 (2006) 12-20. 
[14] M.M. Meerschaert, C. Tadjeran, Finite difference approximations for fractional advectiondispersion flow equations, Journal of Computational and Applied Mathematics 172 (2004) $65-77$.

[15] D.A. Benson, S.W. Wheatcraft, M.M. Meerschaert, The fractional order governing equation of Lévy motion, Water Resources Research 36 (2000) 1413-1423.

[16] A.A. Kilbas, H.M. Srivastava, J.J. Trujillo, Theory and Applications of Fractional Differential equations, Elsevier, 2006.

[17] I. Podlubny, Fractional Differential Equations, Academic Press, San Diego, 1999.

[18] S.G. Samko, A.A. Kilbas, O.I. Marichev, Fractional Integrals and derivatives: theory and Applications, Gordon and Breach Science Publishers, 1993.

[19] P.D. Lax, B. Wendroff, Difference schemes for hyperbolic equations with high order of accuracy, Communications on Pure and Applied Mathematics 17 (1964) 381-398.

[20] E. Sousa, The controversial stability analysis, Applied Mathematics and Computation 145 (2003) 777-794.

[21] K.W. Morton, Numerical solution of convection diffusion problems, Chapman and Hall, London, 1996.

[22] F.F. Warming and B.J. Hyett, The modified equation approach to the stability and accuracy analysis of finite difference methods, Journal of Computational Physics 14 (1974) 159-179.

[23] G.A. Sod, Numerical methods in fluid dynamics: initial and initial boundary-value problems, Cambridge University Press, Cambridge, 1988.

[24] J.H. McCulloch, D.P. Panton, Precise tabulation of the maximally-skewed stable distributions and densities, Computational Statistics \& Data Analysis 23 (1997) 307-320.

[25] A. Ogata, R.B. Banks, A solution of the differential equation of longitudinal dispersion in porous media, U.S. Geol. Surv., Prof. Pap. No. 411-A (1961) A1-A7.

[26] C. Hirsch, Numerical computations of internal and external flows, Vol I and Vol II, WileyInterscience, Chichester, 1990.

[27] G.D. Smith, Numerical solution of partial differential equations, Oxford University Press, Oxford, 1985.

ERcília Sousa

CMUC, Department of Mathematics, University of Coimbra, 3001-454 Coimbra, Portugal 